\title{
Early experience of vascular endothelial growth factor (VEGF) inhibitor in COVID-19 ARDS
}

\section{To the Editor}

The current severe acute respiratory syndrome-corona-virus-2 (SARS-CoV-2) has spread like wildfire and engulfed the entire world in its wake. It has caused immense morbidity and mortality in our country. It causes severe inflammatory response which causes profound pulmonary vasculature endothelial damage and edema leading to generalised hypoxia [1]. This generalised hypoxia causes release of various cytokines including vascular endothelial growth factor (VEGF) through activation of the Prolyl hydroxylases (PHD)-hypoxia-inducible factor (HIF)-1 pathway, which upregulates VEGF expression through transcription activation. VEGF further aggravates the pulmonary edema by increasing the vascular permeability which increases the plasma extravasation [2, 3]. VEGF also has a role in increasing pulmonary inflammation. Though there have been commendable efforts worldwide but there are still no defined treatment modalities for treatment of COVID-19 patients. VEGF inhibitor is being used to treat severe Coronavirus disease 2019 (COVID-19) patients with ARDS with an aim to improve oxygenation, reduce inflammation and improve clinical outcome [1,4]. Herein we report our experience of using VEGF inhibitor - Bevacizumab in three patients at a tertiary care hospital.

All the three patients had confirmed COVID-19 on throat swab reverse transcription-polymerase chain reaction. One of the patients had hypertension and diabetes mellitus as co-morbidities while the other two did not have any other risk factors. Two of the patients were received on Non rebreathing mask (12-15 L/min) and one was maintaining saturation with oxygen supplementation with face mask (6-8 L/min). All the three patients later progressed to severe COVID-19 infection with ARDS requiring non-invasive/invasive ventilation. One of the patients had reported after two weeks of onset of symptom while the rest two presented during the second week. All these patients had raised markers of hyperinflammation such as raised neutrophil/lymphocyte ratio, serum D-dimer, ferritin, LDH and CRP levels (Table 1). These patients received standard institutional care as per the existing guidelines in the form of antipyretics, steroids, therapeutic dose of anticoagulation (enoxaparin $1 \mathrm{mg} / \mathrm{kg}$ subcutaneous twice daily), and awake proning protocol. These patients were diagnosed to have cytokine storm syndrome based on their clinical deterioration and laboratory parameters. One of the patients was also administered Inj Tocilizumab ( $8 \mathrm{mg} / \mathrm{kg}$ ) infusion over 1 hour. However, there was no improvement in clinical or oxygenation status. These patients were then administered Inj Bevacizumab $(7.5 \mathrm{mg} / \mathrm{kg}$ ) infusion over 1 hour. Despite that these patients showed worsening of their clinical condition requiring invasive ventilation and later succumbed to their illness. The pre and post arterial blood gas after 24 and 48 hours did not show much improvement and these patients continued to have high oxygen requirement (Table 2). One of the patients also developed spontaneous haemoptysis as a complication to the anti VEGF therapy. The chest radiograph after 7 days showed sub optimal clearing of opacities (Figure 1A-D). 
Table 1. Demographic profile of patients

\begin{tabular}{|c|c|c|c|c|c|c|c|}
\hline & Age & Sex & Comorbidities & $\begin{array}{l}\text { Day of onset } \\
\text { on presentation }\end{array}$ & $\begin{array}{l}\text { Day of receiving } \\
\text { Bevacizumab }\end{array}$ & $\begin{array}{c}\text { Arterial blood gas } \\
\text { before administering } \\
\left(\mathrm{pO}_{2} / \mathrm{FiO}_{2}\right)\end{array}$ & $\begin{array}{c}\text { ABG after } \\
48 \text { hours } \\
\left(\mathrm{pO}_{2} / \mathrm{FiO}_{2}\right)\end{array}$ \\
\hline Patient 1 & 73 & M & $\begin{array}{c}\text { Type II diabetes, } \\
\text { hypertension }\end{array}$ & 17 & 19 & 150 & 148 \\
\hline Patient 2 & 49 & M & Nil & 12 & 14 & 43 & 52 \\
\hline Patient 3 & 47 & $M$ & Nil & 13 & 14 & 120 & 100 \\
\hline
\end{tabular}

Table 2. Lab parameters of the patients

\begin{tabular}{lcccccc}
\hline & \multicolumn{2}{c}{ Patient 1 } & \multicolumn{2}{c}{ Patient 2 } & \multicolumn{2}{c}{ Patient 3 } \\
\hline & $09 / 04$ & $19 / 04$ & $19 / 04$ & $27 / 04$ & $22 / 04$ & $28 / 7$ \\
$\mathrm{Hb}[\mathrm{g} / \mathrm{dL}]$ & 12.7 & 15.9 & 11.8 & 12.4 & 10.2 & 15.4 \\
$\mathrm{TLC}[\mathrm{cmm}]$ & 10200 & 26700 & 4500 & 14600 & 14000 & 25100 \\
$\mathrm{~N} / \mathrm{L}$ & $87 / 10$ & $93 / 03$ & $93 / 02$ & $92 / 05$ & $84 / 06$ & $85 / 13 / 01 / 01$ \\
$\mathrm{PLT}$ [cmm] & 166000 & 121000 & 195000 & 183000 & 127000 & 129000 \\
PT/INR/PTTK & $14 / 1.0 / 59.1$ & $14.8 / 1.06 / 35.5$ & $15.9 / 1.08 / 39.7$ & $15.1 / 2.29$ & $15.6 / 1.05 / 30$ & $17.2 / 1.34 / 29$ \\
UREA [mg/dl] & 34 & 11 & 34 & 59 & 73 & 89 \\
CREAT [mg/dL] & 0.8 & 0.2 & 1.1 & 0.7 & 1.3 & 1.5 \\
OT/PT [IU/L] & $88 /$ & $58 / 53$ & $39 / 67$ & $40 / 97$ & $28 / 30$ & $28 / 42$ \\
LDH [IU/L] & 548 & 897 & 424 & 499 & 645 & 686 \\
PROCAL & $<.05$ & 0.05 & $<0.05$ & $<0.05$ & $<0.05$ & 0.03 \\
D.DIMER [mg/dL] & 4.3 & 5 & 2.61 & 8 & 3.34 & 7 \\
S. Ferretin [pg/mL] & 710 & 650 & 900 & 804 & 597 & 984 \\
CRP & 106 & 98 & 138 & 100 & 48 & 24 \\
\hline
\end{tabular}

The current SARS-CoV-2 pandemic has caused unprecedented mortality and morbidity worldwide and lack of confirmed effective therapy poses a therapeutic dilemma for the treating physician. It primarily affects the lung and has serious manifestation of ARDS which manifests as severe hypoxia in these patients. VEGF inhibitor (Bevacizumab) is a recombinant, humanized monoclonal antibody which binds to, and neutralizes, vascular endothelial growth factor (VEGF), and prevents its association with endothelial receptors. VEGF binding initiates angiogenesis (endothelial proliferation and the formation of new blood vessels) and its inhibition of microvascular growth is believed to retard the growth of tissues due to which it has been used in various malignancies. has been shown to improve oxygenation, clinical and laboratory features especially C-reactive protein in Severe COVID-19 patients [1, 4]. However, this was not seen in our patients and the one of the reasons could have been delay in administration of this therapy and it was given as last resort for salvaging these patients. Based on our initial experience, we did not find any added benefit of anti VEGF therapy in severe COVID-19 patients and a larger study is needed to confirm its efficacy.

\section{Conflict of interest}

None declared. 


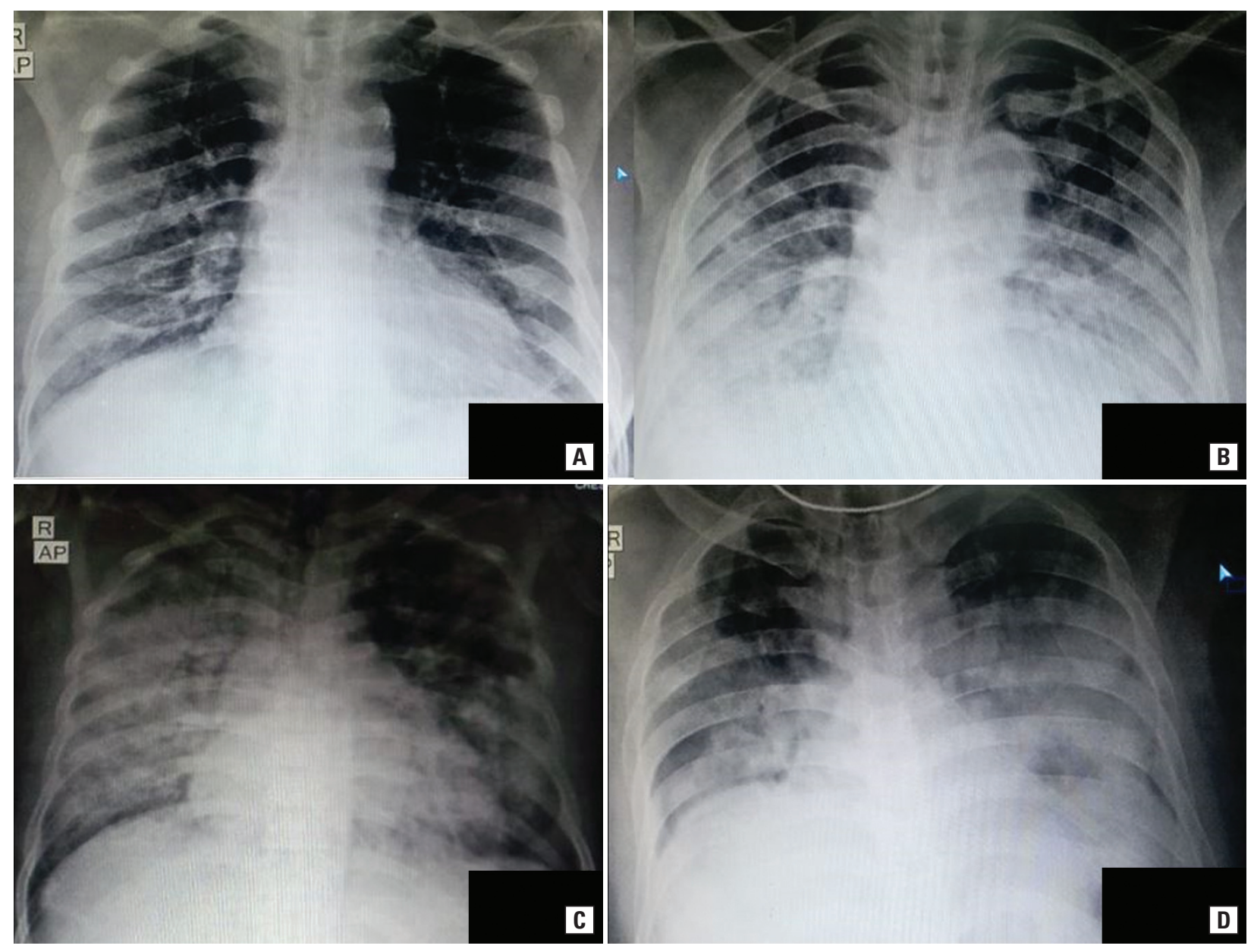

Figure 1. Chest radiograph showing bilateral air space opacities with increased opacities in radiograph $\mathbf{B}$ as compared to $\mathbf{A}$, and $\mathbf{D}$ as compared to $\mathbf{C}$

\section{References:}

1. Pang J, Xu F, Aondio G, et al. Efficacy and tolerability of bevacizumab in patients with severe Covid -19. Nat Commun. 2021; 12, doi: 10.1038/s41467-021-21085-8.

2. Kaner RJ, Ladetto JV, Singh R, et al. Lung overexpression of the vascular endothelial growth factor gene induces pulmonary edema. Am J Respir Cell Mol Biol. 2000; 22(6): 657-664, doi: 10.1165/ajrcmb.22.6.3779, indexed in Pubmed: 10837361.
3. Liu Y, Cox SR, Morita T, et al. Hypoxia regulates vascular endothelial growth factor gene expression in endothelial cells. Identification of a 5' enhancer. Circ Res. 1995; 77(3): 638-643, doi: 10.1161/01.res.77.3.638, indexed in Pubmed: $\underline{7641334}$.

4. Islam MdA, Mazumder MA, Akhter N, et al. Extraordinary survival benefits of severe and critical patients with COVID-19 by immune modulators: the outcome of a clinical trial in Bangladesh. Euroasian J Hepatogastroenterol. 2020; 10(2): 68-75, doi 10.5005/jp-journals-10018-1327, indexed in Pubmed: 33511068. 